\title{
Investigation on Broadening Compressor Surge Margin by Using Active Magnetic Bearing
}

\author{
Yingzhe Lin $\mathbb{D}^{1,},{ }^{1,2}$ Gen Bai $\left(\mathbb{D},{ }^{2}\right.$ Yunkai Huang $\mathbb{D}^{1},{ }^{1}$ Jin Zhou $\mathbb{D}^{3},{ }^{3}$ Xudong Guan $\left(\mathbb{D},{ }^{4}\right.$ \\ and Jiyong Dong $\mathbb{C}^{2}$ \\ ${ }^{1}$ School of Electrical Engineering, Southeast University, Nanjing, China \\ ${ }^{2}$ Nanjing CIGU Limited Corporation, Nanjing, China \\ ${ }^{3}$ College of Mechanical and Electrical Engineering, Nanjing University of Aeronautics and Astronautics (NUAA), \\ Nanjing 210016, China \\ ${ }^{4}$ School of Mechanical Engineering and Rail Transit, Changzhou University, Changzhou 213164, China \\ Correspondence should be addressed to Yingzhe Lin; lyz@cigu.org.cn
}

Received 15 September 2021; Revised 29 January 2022; Accepted 1 February 2022; Published 18 February 2022

Academic Editor: Antonio Giuffrida

Copyright (c) 2022 Yingzhe Lin et al. This is an open access article distributed under the Creative Commons Attribution License, which permits unrestricted use, distribution, and reproduction in any medium, provided the original work is properly cited.

\begin{abstract}
The impeller design usually expects to achieve high efficiency and a wide working range while they are influenced by tip clearance in different ways. In this study, a blower with a controllable magnetic suspension bearing was studied. The magnetic bearing enables the impeller to adjust the tip clearance during operation to optimize the efficiency of the blower at the design point and significantly expand the surge margin when it deviates from the design point. The clearance ratio (CR) is defined as the ratio of tip clearance over impeller outlet width. The computational fluid dynamics (CFD) method is used to study the performance of a centrifugal impeller under $\mathrm{CR}=2.61 \% \sim 7.83 \%$ and verified by experiments. The results show that the pressure ratio and efficiency of the impeller at the design point decreased linearly with the increase of the gap. For each $1 \%$ increment in CR, the pressure ratio reduces by 0.02 and the polytropic compression efficiency declines by $0.5 \%$. Conversely, the surge margin increases with the expansion of CR. For every $1 \%$ rise in CR, the surge margin would improve by $7.8 \%$. A control method of changing the reference suspension position according to the optimal efficiency fitting model is proposed. It realizes the real-time control of the axial suspension position of the impeller according to the changes of pressure and flow rate signals to obtain the optimal work efficiency with a broad surge margin. Experimental results show that using an impeller equipped with a controllable magnetic suspension bearing could minimize the gap stably in the nonsurge area to obtain high efficiency, and when necessary, it could enlarge the gap to meet the low flow rate condition.
\end{abstract}

\section{Introduction}

Maglev technology in recent years has brought great innovation for the industry compressor. However, limited by the strength of the material, high-speed impellers are usually unshrouded which leaves a gap between the top of the blade and the side of the cover. For safety reasons, the gap cannot be diminished and the leakage flow caused by the tip gap will affect the efficiency of the impeller, pressure rise, surge margin, etc. Unlike the traditional blower, the controllable magnetic bearing can adjust the blade tip clearance within a certain range so that the magnetic levitation impeller can theoretically achieve better performance in both efficiency and surge margin.
Senoo $[1,2]$ proposed a prediction model for the clearance loss and conducted experimental verification. Their results showed that the tip clearance loss is basically proportional to the clearance ratio (when the clearance ratio is less than 0.1). LIU [3] explored the performance of Eckardt impellers under three different gap values through numerical simulation and experiments. In summary, the tip clearance is inversely correlated with the efficiency and pressure ratio of the impeller. The smaller the clearance is, the higher the efficiency of the impeller could achieve.

Surge is an inevitable problem in turbomachinery. Galindo et al. [4] explored the influence of different gaps near the surge condition. Increasing the tip clearance allows 
a part of the gas to flow back to the inlet through the clearance, which increases the inlet flow and broadens the surge margin. Hewkin-Smith et al. [5] studied the role of axial flow blade tip clearance in the pulse-like rotating stall. An axial compressor was studied and showed that the surge margin increased when CR increased from $0 \%$ to $0.5 \%$. There are other ways to broaden compressor surge margin which includes self-adaptive casing [6] and variable vanes [7]. However, they require extra components and very complicated structure which make them uneconomical for industry applications.

For magnetic levitation blowers, real-time gap adjustment can be achieved through active control, that is, keeping a small gap at the design point to obtain high performance and increasing the tip gap to expand the surge margin when it deviates from the operating point [8]. In this way, it grants the impeller high performance and wide working range simultaneously. Sanadgol and Maslen [9] realized the control of the axial clearance of the impeller by adjusting the axial active magnetic bearing (AMB) system. Yoon et al. [10-12] used axial AMB to adjust the axial clearance of the compressor impeller based on the magnetic levitation compressor test bench to achieve surge control. Aiming at the surge problem of magnetic levitation compressors, Tang et al. [13] designed feedback control strategies based on mass flow rate and output pressure, respectively. The simulation results showed that the adopted control strategy could effectively expand the stable operation range of the compressor. Guan et al. [14] combined the magnetic levitation compressor model and the magnetic bearing rotor test bench to obtain a semiphysical simulation surge control platform and verified the simulation surge control effect under different speeds and reference adjustment trajectories. Sun et al. [15] proposed an online surge detection method by monitoring the axial displacement of the rotor. Ma et al. $[16,17]$ studied the surge suppression technology of the AMB system. The rotor was stabilized under surge condition by using the active-disturbance-rejection controller. Bonfitto et al. $[18,19]$ studied the offset-free model predictive control for AMBs and cone-shaped AMBs. This method not only takes full advantage of the superiorities of classical model predictive control in terms of stability and control performance but also overcomes the influence of model mismatch on reference tracking. In conclusion, the idea that suppressing compressor surge by adjusting tip clearance in blower equipped with the AMB system is not new. However, most of the previous studies are only simulation analysis and their tip control models are too complicated to execute on industry application.

In this article, the performance of a commercial magnetic levitation blower under the control of axial magnetic bearings at different tip clearances and different speeds is studied through experiments and numerical simulations. First, the performance maps of the impeller under different clearances are obtained by the numerical simulation method and verified by experiments. Secondly, according to the experimental data, the minimum gap value under different flow rates and pressures in the near-surge area is determined. The first-order linear regression is adopted to find the control law of the gap value with respect to the flow rate and pressure. Finally, the control method proposed in this paper to change the axial suspension position of the impeller is used to achieve the balance between efficiency and surge margin. Then, the feasibility of the proposed control method is verified through experiments.

\section{The Study Models}

The case studied in this paper is a commercial compressor. Its main flow components include a backward curved impeller with splitter blades, a vaneless diffuser, and a volute. Its design specific speed is 0.793 and maximum pressure ratio and maximum flow rate are 2.1 and $1.1 \mathrm{~kg} / \mathrm{s}$, respectively. The designed static installation gap is $0.4 \mathrm{~mm}$ and the meridian width of the vaneless diffuser with the pinch is $10.37 \mathrm{~mm}$. See Figure 1 and Table 1, for detailed geometric parameters.

\section{The Correlation between Surge Margin and Tip Clearance}

3.1. The CFD Study. The expression of surge margin S [20] and clearance ratio (CR) are listed below:

$$
\begin{aligned}
S & =\left(\frac{\pi_{s} / m_{s}}{\pi_{d} / m_{d}}-1\right) \times 100 \%, \\
C R & =\frac{c}{b} \times 100 \%,
\end{aligned}
$$

where $\pi_{s}$ is the pressure ratio at surge point , $m_{s}$ is the surge flow rate,$\pi_{d}$ is the pressure ratio at design point, and $m_{d}$ is the design flow rate. The CR (clearance ratio) is defined as the ratio of tip clearance $c$ over blade outlet width $b$.

To obtain the relationship between surge margin $S$ and $\mathrm{CR}$, the performance maps of the compressor were calculated by CFD, as shown in Figure 2. Besides the pressure ratio, the polytropic efficiency obtained from CFD is also plotted to compare with the experimental data.

The commercial CFD software CFX was used to simulate the flow field of the impeller, the vaneless diffuser, and the volute. To reduce the demand for computing resources, the impeller and diffuser adopt a single-channel periodic boundary condition. The frozen rotor model was used to connect the rotating and stationary parts. The impeller was divided by structural grids with 15 layers of grids in the blade tip gap along the blade height direction to capture the gap flow. The volute was generated by a nonstructural grid and connected with the vaneless diffuser by the frozen rotor model.

Numerical simulations calculated the performance map with CR which varied from $2.61 \%$ to $7.83 \%$. Besides, the performance maps when CR was $2.61 \%, 4.35 \%$, and $6.09 \%$ were verified by experiments.

In CFD study, the surge point was determined by gradually reducing the outlet flow at the same speed. When the outlet pressure no longer increased with the decrease of the flow or the calculation showed that the outlet pressure did not converge over iterations, the point was considered to be surge. 


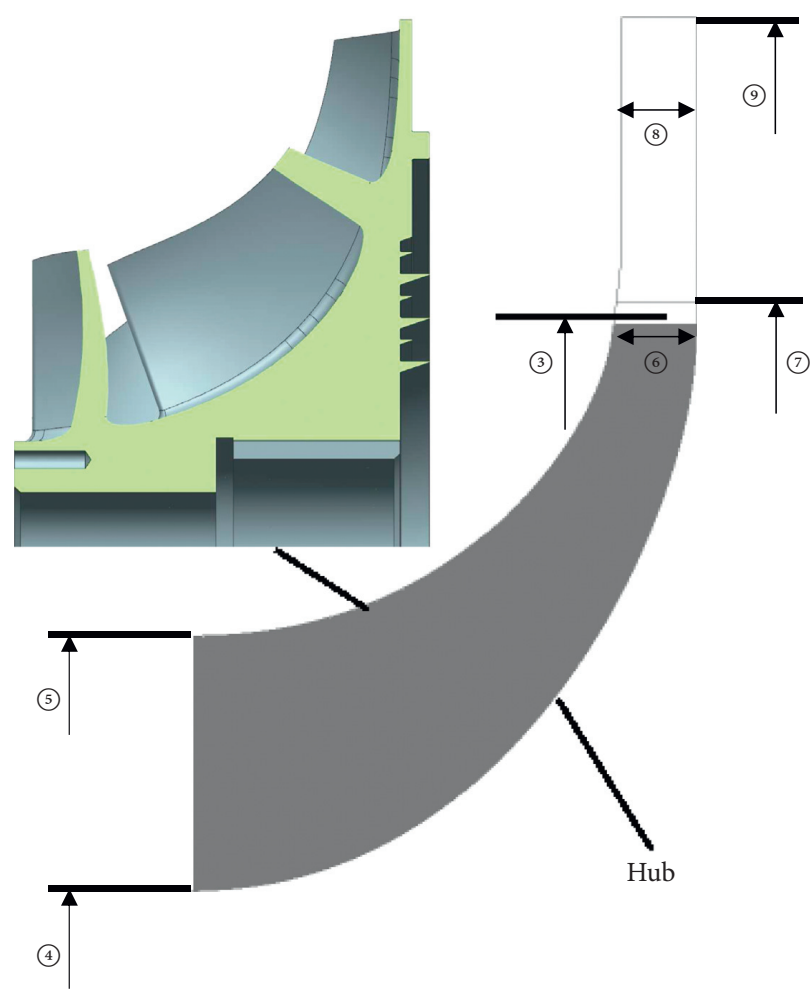

(a)

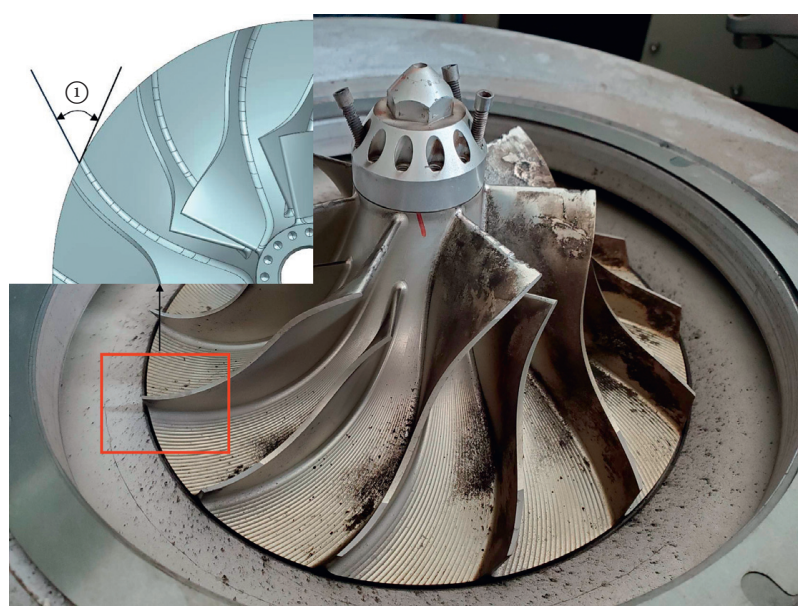

Figure 1: Test impeller. (a) Meridional sketch. (b) Test prototype.

TABLE 1: Geometrical parameters.

\begin{tabular}{lclc}
\hline \multicolumn{1}{c}{ Impeller } & & Diffuser \\
\hline (1) Number of blades & 14 & (7) Pinch out radius & $112.125 \mathrm{~mm}$ \\
(2) Blade backswept angle & $38.5^{\circ}$ & (8) Constant height & $10.37 \mathrm{~mm}$ \\
(3) Exit radius & $97.5 \mathrm{~mm}$ & (9) Exit radius & $170 \mathrm{~mm}$ \\
(4) Inlet hub radius & $20.3 \mathrm{~mm}$ & & \\
(5) Inlet shroud radius & $55 \mathrm{~mm}$ & & \\
(6) Exit blade height & $11.52 \mathrm{~mm}$ & & \\
\hline
\end{tabular}

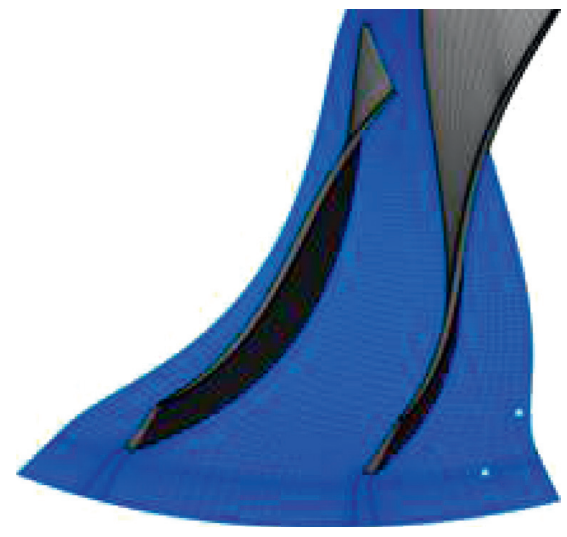

(a)

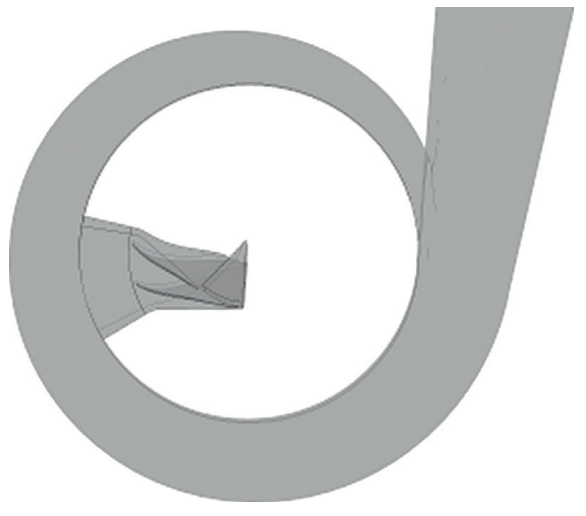

(b)

Figure 2: Computational mesh of the impeller and the entire model. (a) Impeller single-channel mesh. (b) The overall CFD model. 
Figure 3 shows the performance maps of the impeller obtained through CFD in all operating conditions and the trend of pressure and efficiency at the design point as the CR increases. Generally speaking, as the CR increases, the pressure ratio over the flow rate curve moves to the lower left and the compression efficiency declines. In the studied CR range, the pressure ratio at different speeds drops with the rise of CR at different speeds. At $100 \%$ speed, the pressure ratio drops by about 0.02 for every $1 \%$ increase in CR; at $90 \%$ speed, it decreases by 0.011 ; at $80 \%$ speed, it declines by 0.0098 . The decrease rates of efficiency with increasing CR at different speeds are relatively close. For every $1 \%$ increase in $\mathrm{CR}$, the efficiency decreases by about $0.5 \%$.

3.2. The Tip Clearance Effects on Surge Margin. By definition of $S$, Figure 4 shows the relationship between surge margin and CR. The design point flow rate and pressure ratio are $1.1 \mathrm{~kg} / \mathrm{s}$ and 1.905 individually. The surge margin at different speeds all increase with the rise of CR and alter approximately linear. The surge margin rises from $26 \%$ to $59 \%$ as CR varies from 0.026 to 0.07 at $32000 \mathrm{r} / \mathrm{min}$. In addition, compared with the decrease in efficiency, the surge margin increases faster with the increase of CR. For every $1 \%$ increase in CR, the surge margin will increase by about $7.8 \%$.

Figure 5 shows the meridional velocity contour under near-surge conditions with different CR. It can be seen that, as CR increases, the low-velocity zone at the shroud side gradually increases and the affected area spreads from the rear to the inlet. The gap flow under the large CR squeezes the separation at the leading edge due to the reduced flow rate so that the impeller can work at a lower flow rate. In addition, the larger gap also allows the compressed highenergy gas in the rear section to reinject to the front edge to increase the energy of the inlet airflow and broaden the surge margin.

3.3. Clearance Control Model. Following the preceding discussion, increasing the tip clearance can broaden the surge margin, but at the same time, it will deteriorate the efficiency. Usually, when the machine is running, it is expected that the efficiency is as high as possible while adapting to different working loads. Based on this, a clearance control method is proposed that when the compressor is working at the nonsurge condition, the tip clearance is fixed at the minimum gap through the magnetic bearing to gain the highest efficiency; when entering the surge region, according to the corresponding load conditions, the gap is enlarged to the minimum workable gap where no surge occurs.

In order to simplify the problem, only the situation at the rated speed is considered. Therefore, it can be considered that the optimal gap value under different working conditions is only related to the current flow rate and pressure rise. The volume flow rate and pressure rise at surge point under different clearances were calculated by varying the boundary conditions and clearance values of the mesh in CFD. The results are shown in Table 2.
Based on the data of Table 2, the two-region control law which keeps constant clearance at normal flow rate and adjust clearance at near-surge flow rate is plotted in Figure 6.

According to the data in Table 2, the linear regression equation is obtained; then, the gap value $C=f(P, Q)$, where $Q$ is the volume flow rate and $\mathrm{P}$ represents pressure rise:

$$
C=-0.04051 Q-0.01713 P+3.7341 \text {. }
$$

The regression error of the equation is shown in Table 3. It can be seen that the maximum error of the first-order linear regression is $0.0293 \mathrm{~mm}$.

Therefore, at the speed of $32000 \mathrm{r} / \mathrm{min}$, the optimal clearance could be calculated through equation (3) as given below:

$$
C(P, Q)=\left\{\begin{array}{l}
-0.04051 \times Q-0.01713 \times P+3.7341, Q<45 \\
0.3, Q \geq 45
\end{array},\right.
$$

where $0.3 \mathrm{~mm}$ is the minimum clearance that the AMB system allowed.

\section{The AMB Control System}

The basic dimension and assembly relationship of the rotor are shown in Figure 7. Each radial magnetic bearing is equipped with a displacement sensor. The detailed information of the AMB is listed in Table 4. The mass of the rotor and impeller is $8.7 \mathrm{~kg}$ and $0.91 \mathrm{~kg}$, respectively.

The schematic diagram of each component of the magnetic bearing system and its working principle is shown in Figure 8. It is mainly consisted of a controller, a power amplifier, magnetic bearings, sensors, and the controlled object. In an ideal situation without external interference, the resultant electromagnetic force generated by the current of the winding in the magnetic suspension bearing is equal to the gravity of the controlled object. Thus, stable suspension can be achieved without control. However, in the actual magnetic levitation system, the open-loop magnetic levitation system is unstable due to the negative displacement stiffness of the electromagnetic force, so closed-loop control is required. Specifically, when the rotor is disturbed by the outside disturbance, it will deviate from the original stable suspension position. The system detects the offset of the controlled object through the displacement sensor and feeds it back to the controller. The controller outputs the control voltage signal to the power amplifier after calculation according to the designed control law. The power amplifier converts the input control voltage signal into the control current in the winding and generates the corresponding control electromagnetic force so that the deviated controlled object returns to the original equilibrium position.

To realize the economic operation of the blower, the axial bearing is controlled as the actuator to alter the impeller axial position. Figure 9 shows the flowchart of the variable impeller suspension position control. The PID control method is adopted for the AMBs. When the pressure and flow rate signal changes, the controller adjusts the reference suspension position according to the optimal efficiency fitting model to realize the real-time change of the axial suspension 

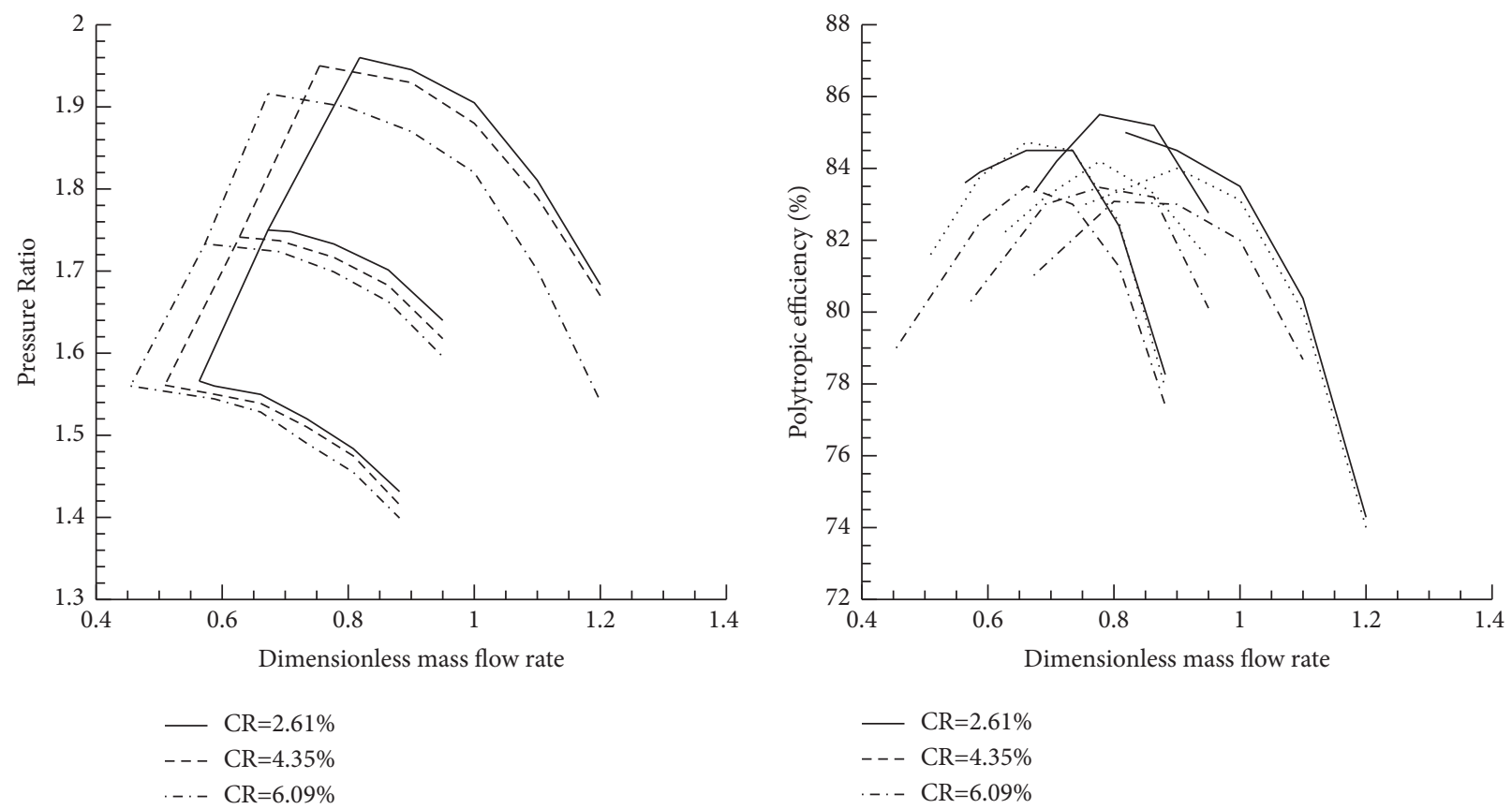

(a)

(b)

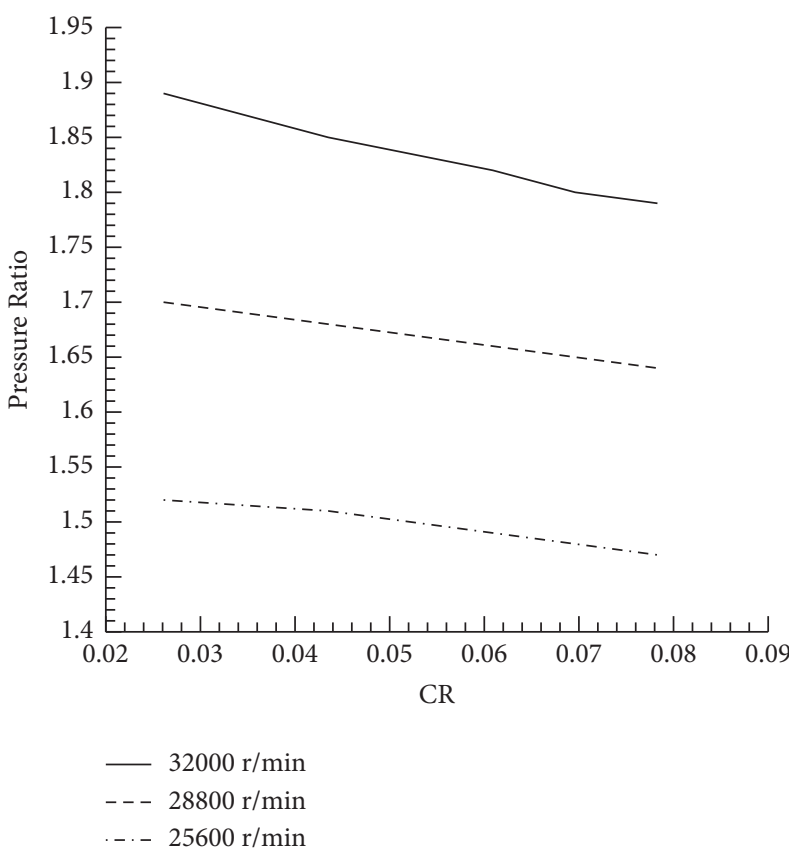

(c)

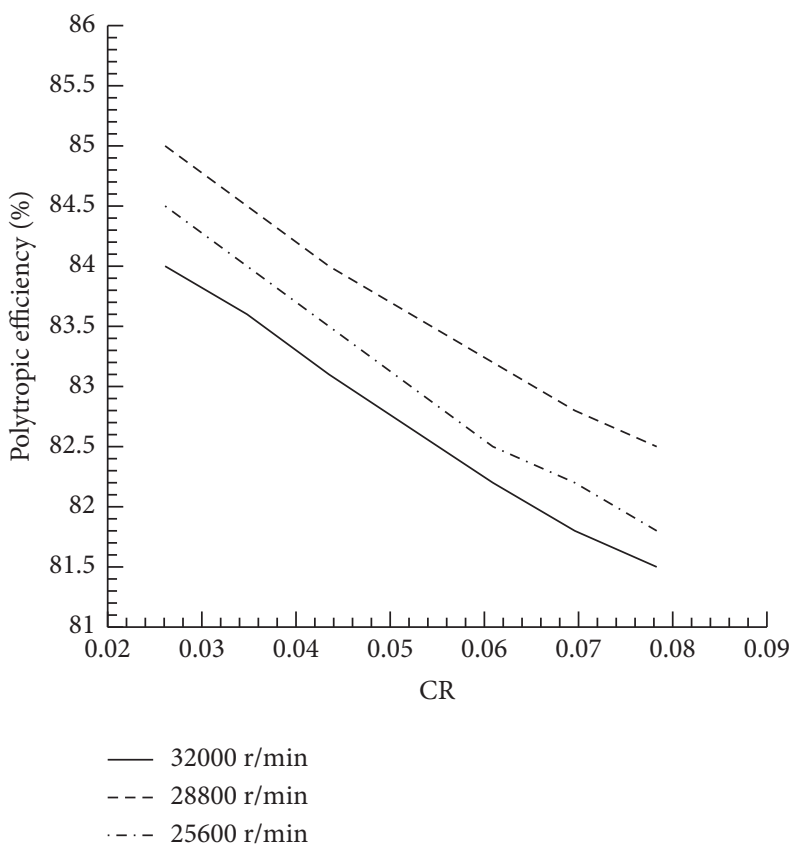

(d)

FIGURE 3: Overall performance maps of pressure ratio and efficiency over CR. (a) Pressure ratio over mass flow rate. (b) Polytropic efficiency versus mass flow rate. (c) Pressure ratio over clearance ratio. (d) Polytropic efficiency over clearance ratio.

position of the impeller. Thus, the impeller could always achieve the optimal efficiency without surging.

\section{Experimental Verification}

5.1. The Performance Map Experiment. As shown in Figure 10, the experimental device composed a commercial CIGU magnetic levitation centrifugal blower, installed a collector in front of the impeller inlet. Besides, temperature and pressure probes were fixed on the inlet and outlet pipes to record the operating status of the machine under different tip clearances. The axial magnetic bearing control was executed by connecting the power amplifier board and the digital-to-analogy converter to the computer. The impeller static clearance was $0.5 \mathrm{~mm}$ when installed. The magnetic levitation controller was used to adjust the rotor position so that the clearance varied from $0.3 \mathrm{~mm}$ to $0.7 \mathrm{~mm}$ during the operation. 


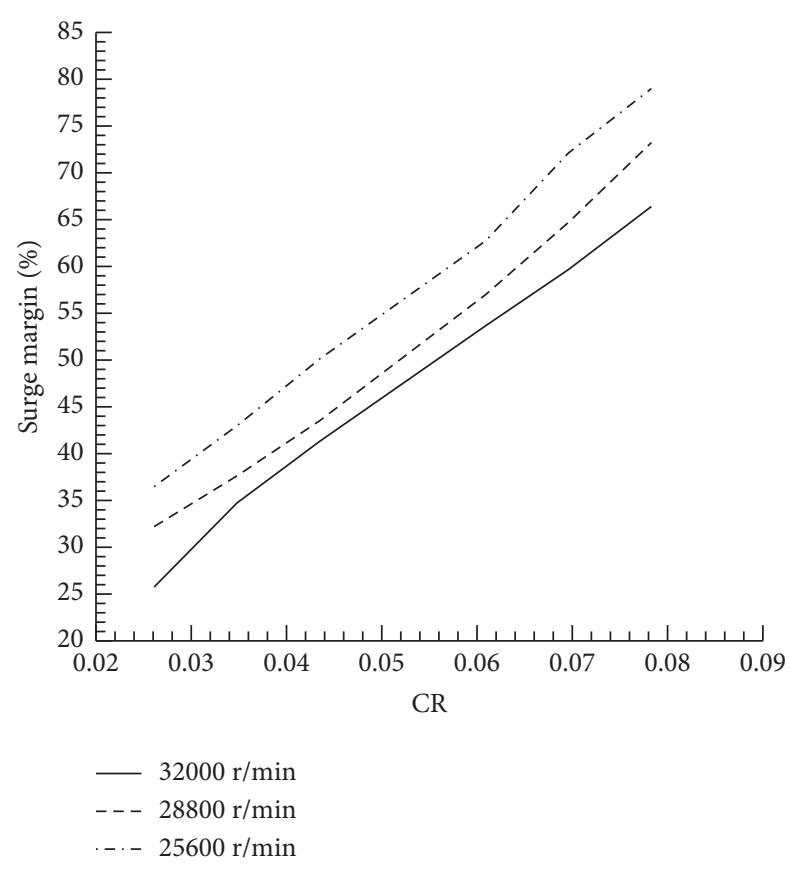

FIGURE 4: Surge margin over CR.

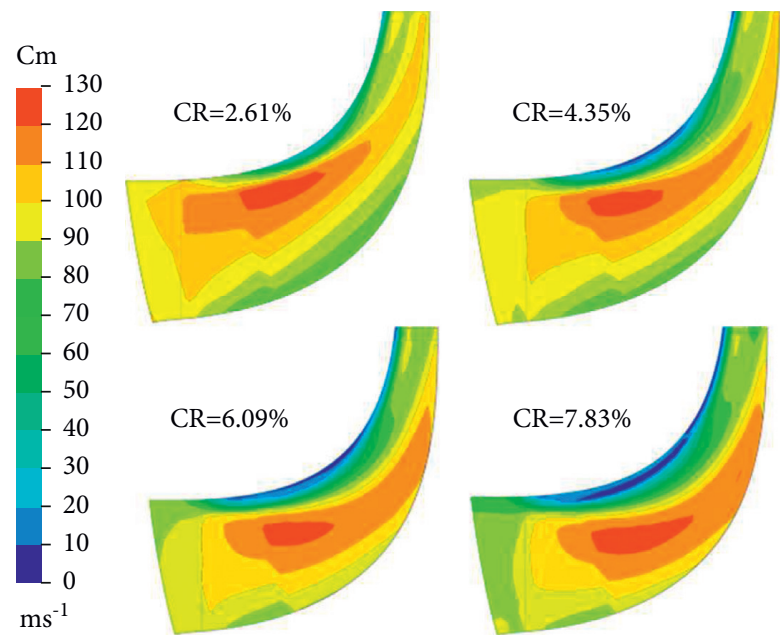

FIgURe 5: Meridional velocity contour at different CRs.

TABLE 2: The flow rate and pressure rise of surge point under different clearances.

\begin{tabular}{lcc}
\hline Volume flow rate $\left(\mathrm{m}^{3} / \mathrm{min}\right)$ & Pressure rise $(\mathrm{kPa})$ & Clearance value $(\mathrm{mm})$ \\
\hline 45.00 & 94.7 & 0.3 \\
42.00 & 94.9 & 0.4 \\
40.00 & 94.5 & 0.5 \\
36.00 & 90.3 & 0.7 \\
34.50 & 89.5 & 0.8 \\
33.00 & 88 & 0.9 \\
\hline
\end{tabular}

The experiment obtained the efficiency of the whole machine by measuring the temperature and pressure of the inlet and outlet. The flow rate was obtained from the static pressure in the inlet venturi. For the determination of surge, the outlet pipeline was equipped with a precision pressure gauge. When the outlet pressure had obvious periodic oscillations, it was recorded as the surge point at the current speed.

It can be seen from Figure 11 that the CFD results are more consistent with the experimental results in terms of 


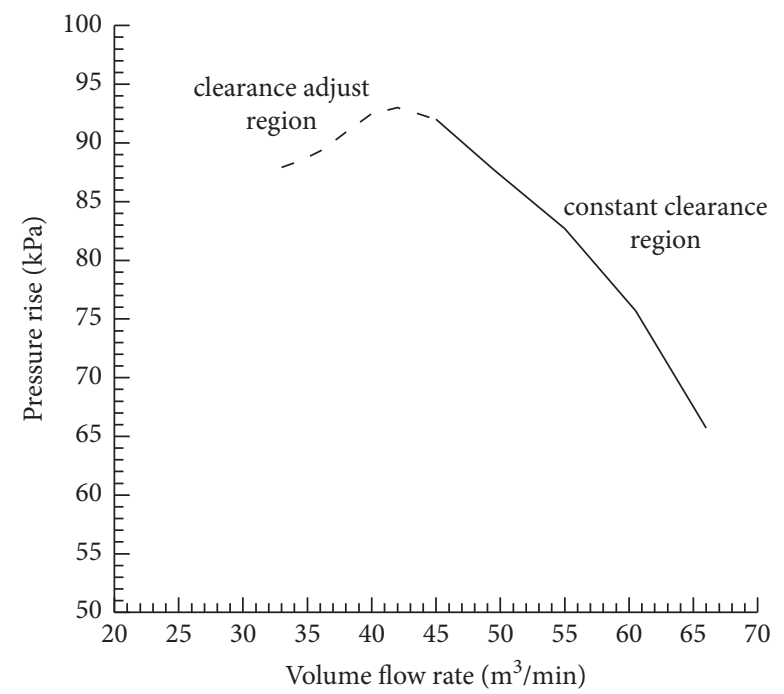

FIgURE 6: $32000 \mathrm{r} / \mathrm{min}$ constant and variable clearance region.

TABle 3: Errors of the equation.

\begin{tabular}{lccc}
\hline No. & Prediction C $(\mathrm{mm})$ & Residual & Standard residual \\
\hline 1 & 0.289341 & 0.010659 & 0.5783769 \\
2 & 0.407452 & -0.00745 & -0.404370 \\
3 & 0.495326 & 0.004674 & 0.2536037 \\
4 & 0.729300 & -0.02930 & -1.589896 \\
5 & 0.803768 & -0.00377 & -0.204487 \\
6 & 0.874812 & 0.025188 & 1.3667722 \\
\hline
\end{tabular}

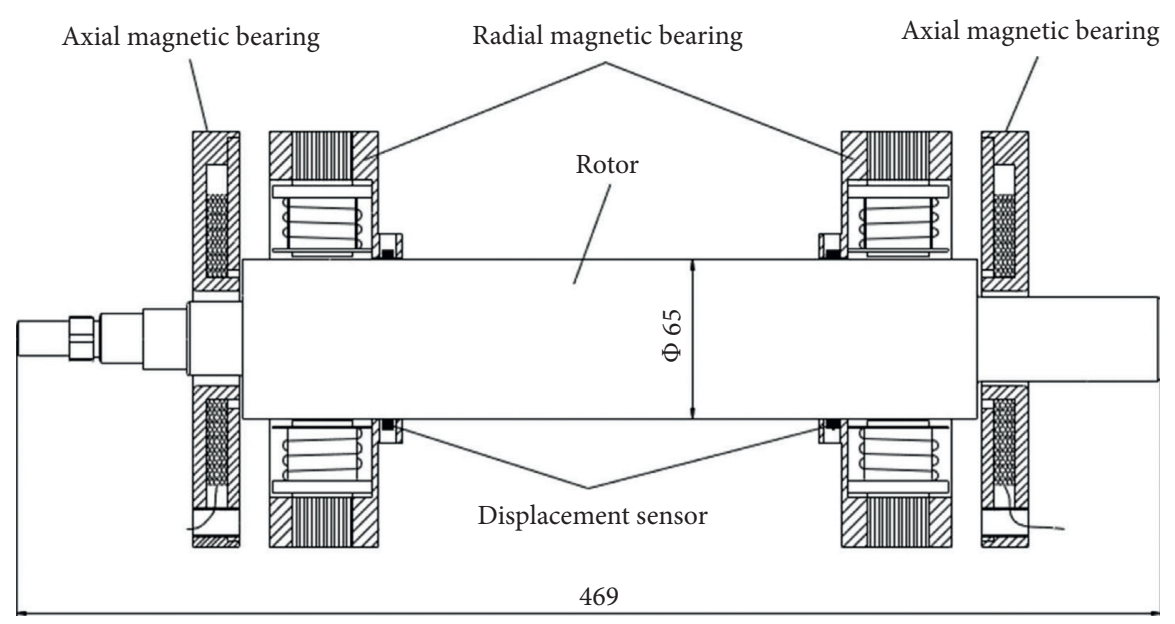

FIgURE 7: The sketch of the rotor.

TAble 4: Parameters of the AMB.

\begin{tabular}{lcccc}
\hline Item & Symbol & Radial AMB & Axial AMB & Unit \\
\hline Vacuum permeability & $\mu_{0}$ & $4 \pi \times 10^{-7}$ & $4 \pi \times 10^{-7}$ & $\mathrm{~N}^{2} \mathrm{~A}^{2}$ \\
Pole area & $A$ & 375 & 768.8 & $\mathrm{~mm}^{2}$ \\
Coil turns & $N$ & 148 & 240 & 2.5 \\
Bias current & $I_{0}$ & 2.5 & $\mathrm{~A}$ \\
\hline
\end{tabular}




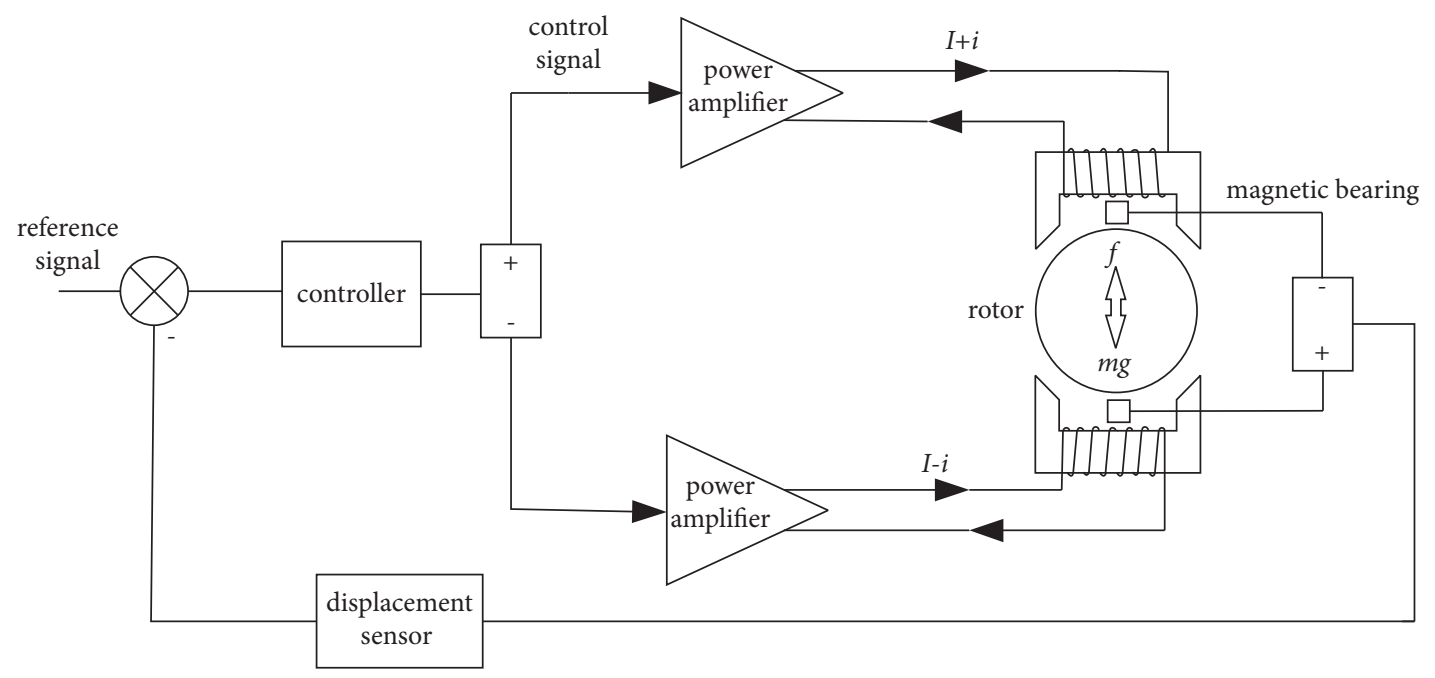

FIGURE 8: Magnetic bearing system.

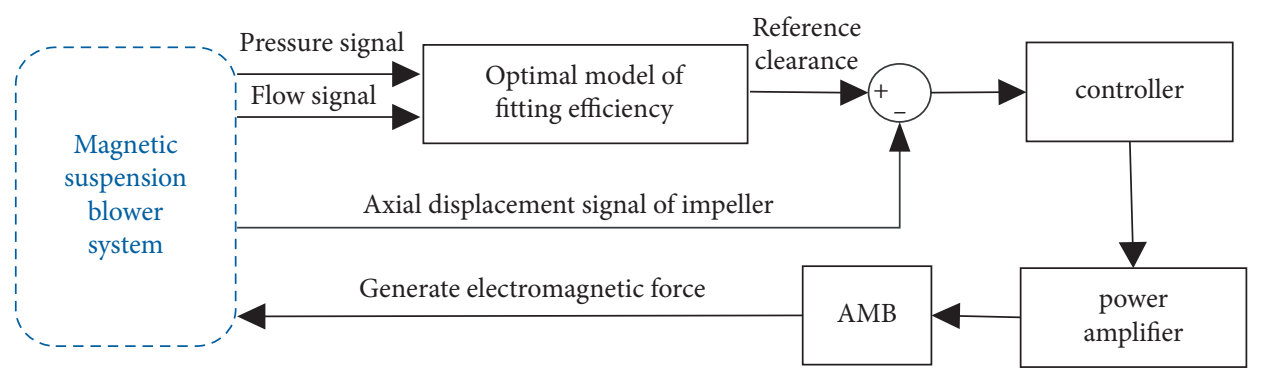

FIGURE 9: Impeller suspension position control flowchart.

overall performance indicators. In the calculation of the pressure ratio, the maximum deviation between CFD and the experiment is $1.8 \%$; as for the polytropic efficiency, CFD is overestimated by about $1 \%$. This is mainly due to the deviation of the adiabatic wall assumed in the calculation and the fact that friction loss, leakage loss, and some transient losses could not be captured through steady-state calculations. The specific trend is very similar between the CFD and experimental data. In addition, the relatively large difference between CFD and experimental data at high speed is mainly due to the failure of the CFD model to consider the deformation of the impeller at high speed.

\subsection{Experimental Research on Variable Impeller Axial Suspension Clearance Control}

5.2.1. Test of the Clearance Control Law. In order to reflect the relationship between the axial suspension position of the impeller and the efficiency of the blower, according to formula (3), as shown in Figure 12, the optimal efficiency curve and the change of the reference suspension position of the impeller are fitted with the variation of pressure rise and flow rate. It can be seen from Figure 12 that, as the flow rate increases, the impeller gap gradually decreases, and as the pressure increases, the impeller gap decreases. Therefore, under different conditions, a suitable axial adjustment position of the impeller can be found and real-time control can be carried out according to this law.

According to the optimal impeller suspension position control method in Figure 9, by varying the valve opening, the experiment shows that the impeller suspension position changes with the altering of the flow rate and pressure rise. It can be seen from Figure 13 that, as the axial suspension position of the impeller varies, the outlet pressure and flow rate of the blower also changes. When the impeller levitation gap decreases, the voltage at the axial levitation position sensor of the rotor gradually decreases, the blower pressure rise declines, and the blower inlet flow rate increases. Conversely, as the impeller suspension gap increases, the pressure rise increases and the flow rate decreases. The above experimental results show that the use of the control method proposed in this paper makes the working position of the magnetic levitation blower gradually move from the point close to the surge condition to the stable working region on the characteristic curve.

The trajectories of both ends of the rotor during the axial change of the rotor are shown in Figure 14. $C_{\max }$ in Figure 14 reflects the radial $A M B$ clearance. It can be seen that the trajectory of the rotor displacement is below $0.3 C_{\max }$. Therefore, the compressor could work stably for a long time according to the ISO14839-2 standard [21]. Besides, it shows that the PID method can effectively control the rotor during operation. 


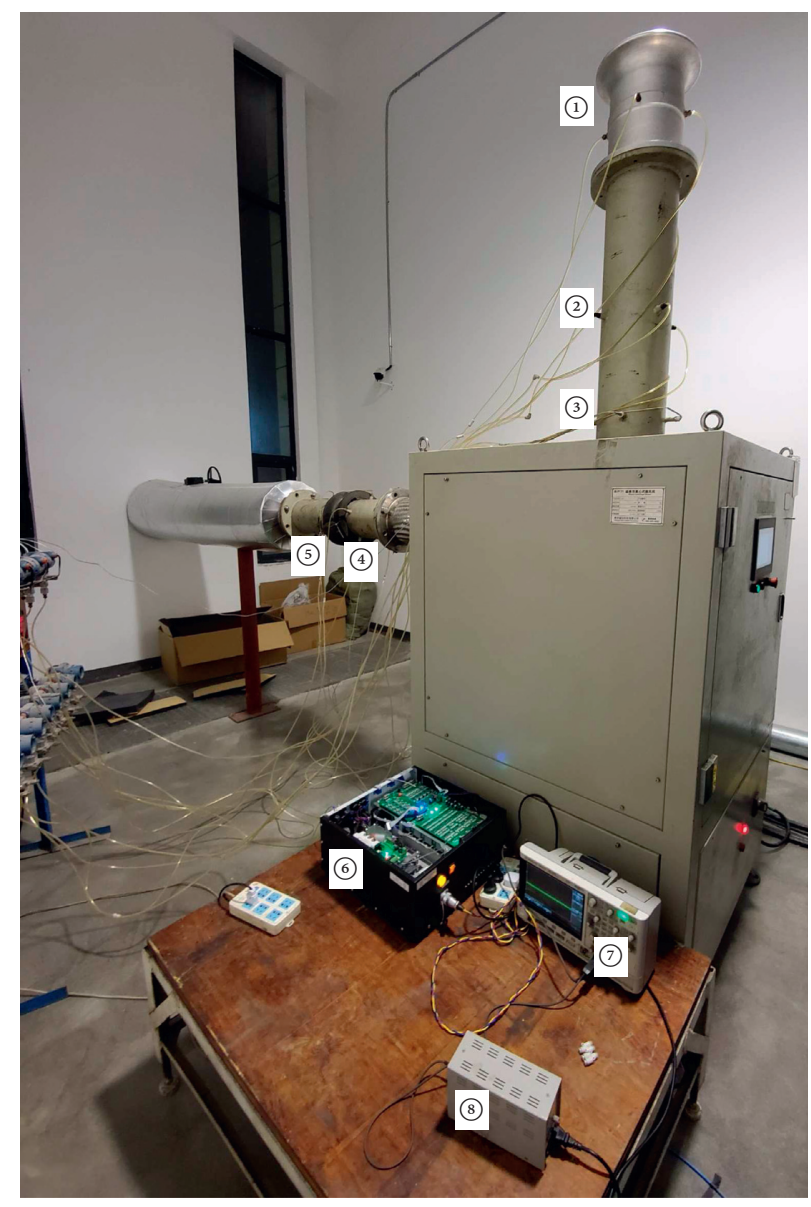

(1). Bell ring (2) \&(4). Pressure gauge (3) \&(5). Temperature sensor (6.AMB power amplifier (7). Oscilloscope (8.DAC

(a)

Figure 10: Continued. 


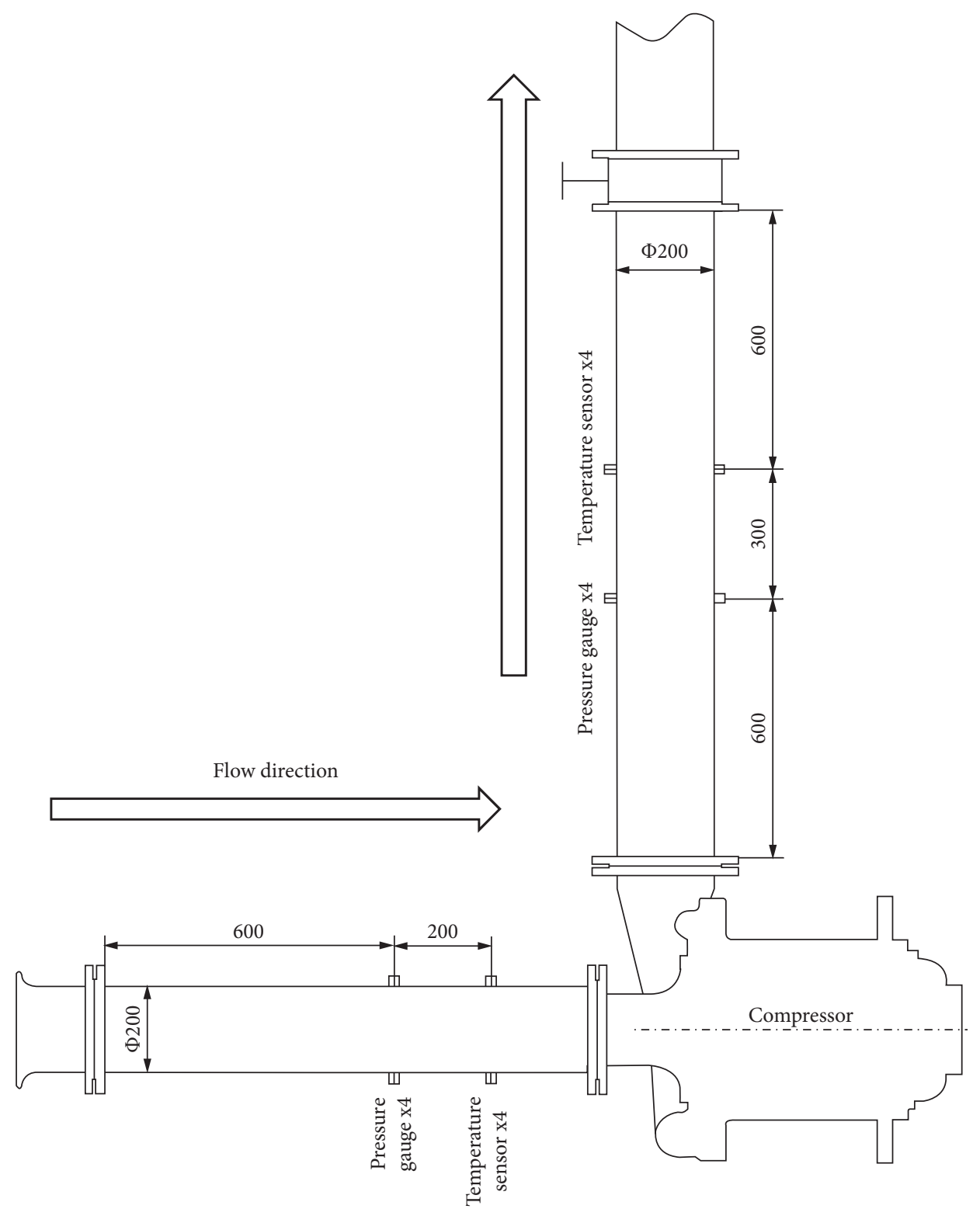

(b)

FIgure 10: Test configuration. (a) Test equipment. (b) Test rig dimensions.

5.2.2. Results on Control Performance Under Surge. The control law was programmed into the magnetic bearing controller. During the test, the speed rose to the rated speed of $32000 \mathrm{r} / \mathrm{min}$, and the throttle valve gradually closed from the maximum opening to the surge flow rate. The flow rate, pressure rise, and efficiency of the compressor were recorded.

Limited by the magnetic bearing structure, the adjustment range of the axial gap in the experiment was only $0.3 \mathrm{~mm}-0.7 \mathrm{~mm}$. It can be seen from Figure 15(a) that, in the fixed gap range, the test pressure ratio is in good agreement with the CFD results. When the throttle valve is gradually close to the surge flow rate, the controller starts following the control law to enlarge the axial clearance so that the blower can work normally in the surge area of the original fixed clearance. Figure 15(b) shows the comparison between the
CFD optimal efficiency curve and the experimental test efficiency. The test efficiency in the fixed gap area is in good agreement with CFD while the test efficiency in the surge control region is $0.5 \%$ lower than the CFD value. The reason is that the steady-state CFD failed to capture the complex flow phenomenon at low flow rate which leads to an overestimate of polytropic efficiency. Besides, to avoid a surge shutdown during the test, the actual gap value was slightly larger than the theoretical optimal value, but the general trend is very consistent. In general, under the test clearance control range of $0.3 \mathrm{~mm}-0.7 \mathrm{~mm}$, the surge margin of the impeller has increased from $28 \%$ to $55 \%$ and the minimum flow rate at which the impeller can work under the rated speed has dropped from $44 \mathrm{~m}^{3} / \mathrm{min}$ to $36 \mathrm{~m}^{3} / \mathrm{min}$ while the polytropic efficiency remains the same. 


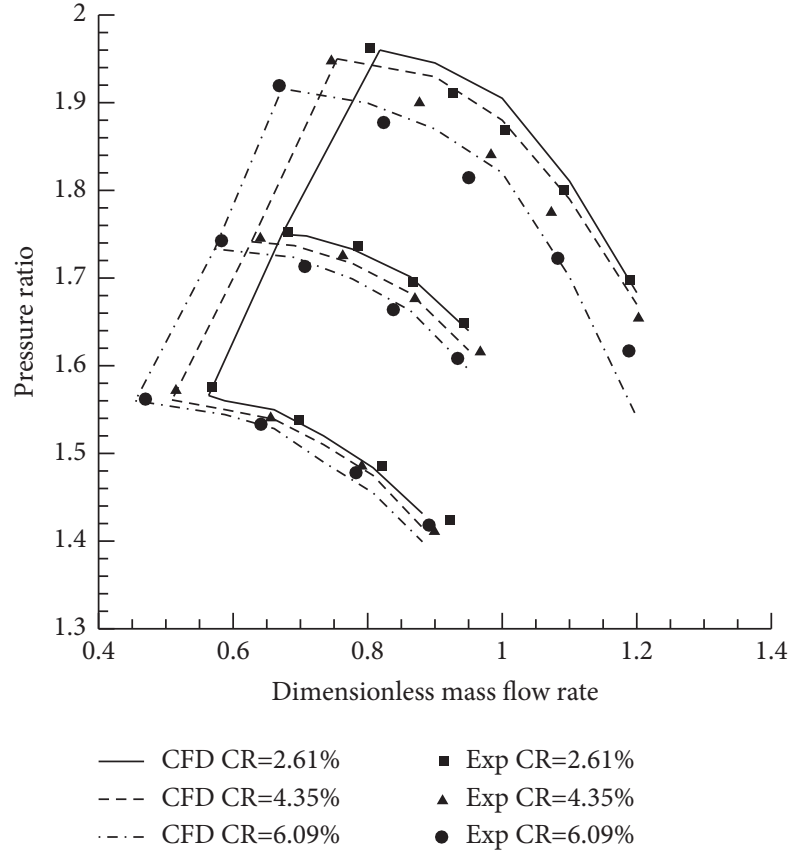

(a)

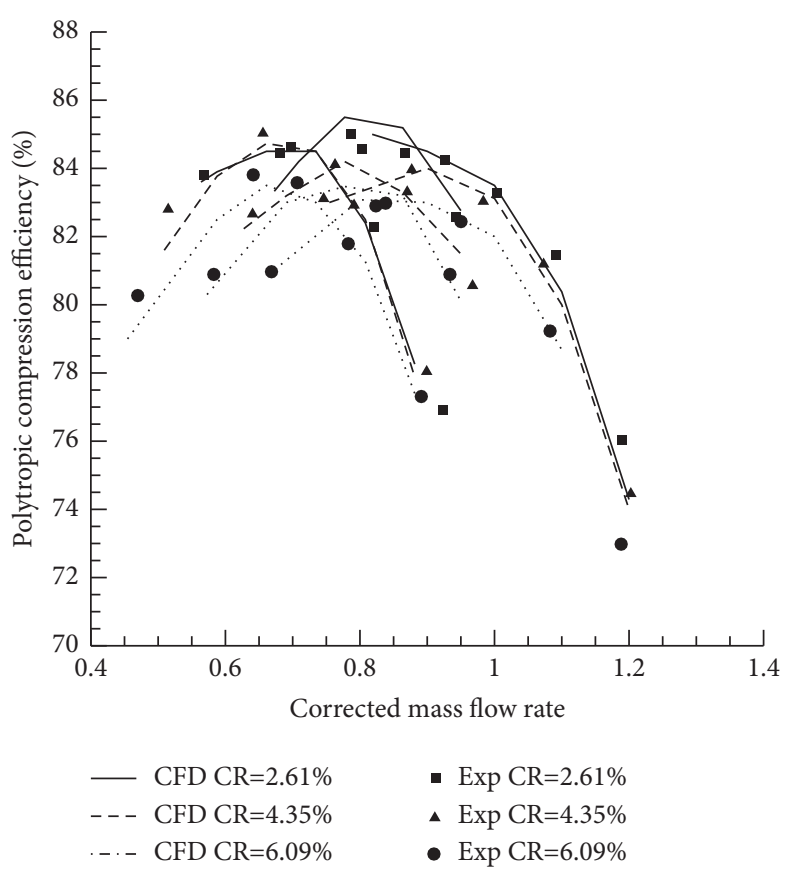

(b)

Figure 11: Comparison of CFD and experimental data. (a) Comparison of pressure ratio over mass flow between CFD and experimental results. (b) Comparison of polytropic efficiency over mass flow between CFD and experimental results.

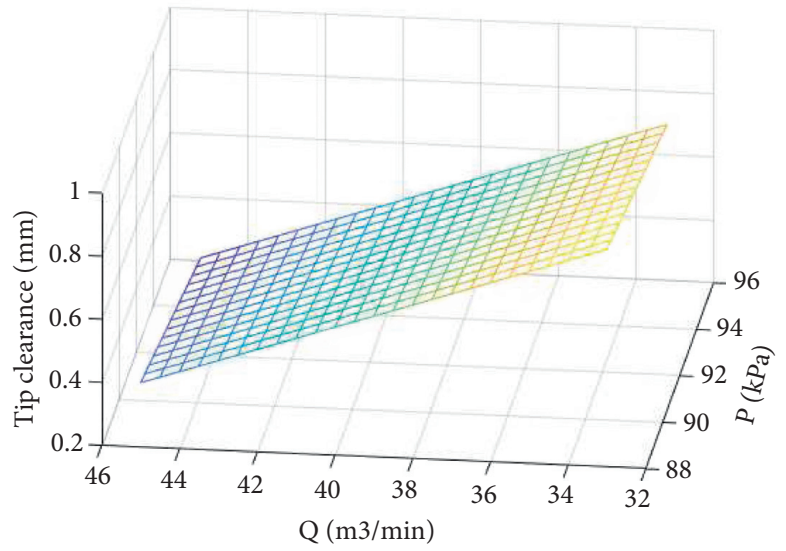

FIgURE 12: The axial clearance variation with flow rate and pressure rise.

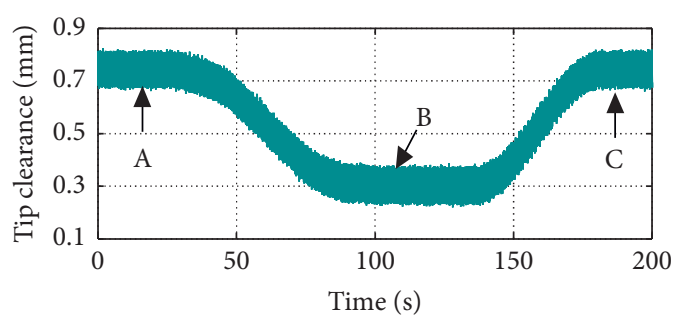

(a)

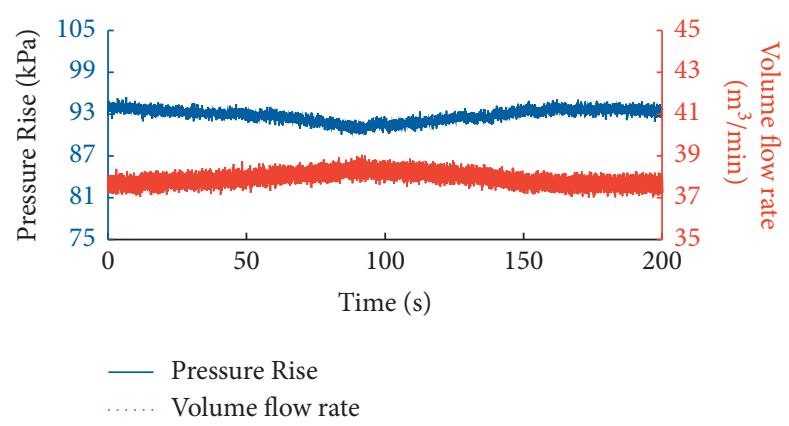

(b)

FIgURE 13: Test results of axial suspension control. (a) Axial displacement signal of impeller. (b) Pressure rise and volume flow rate. 

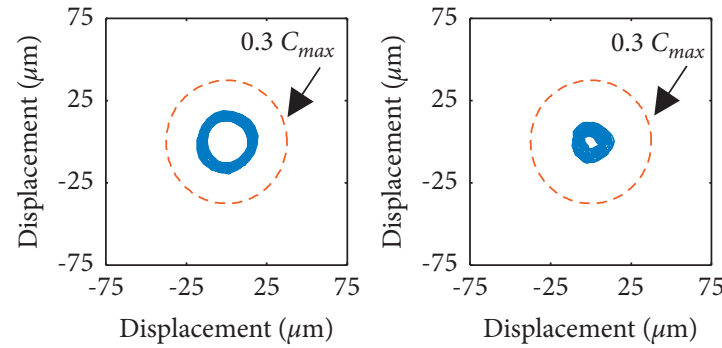

(a)

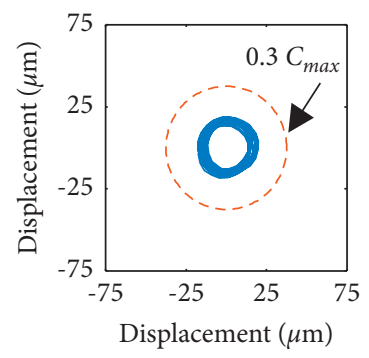

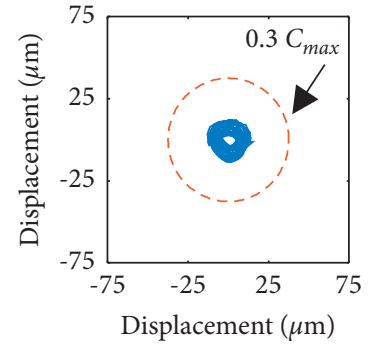

(b)

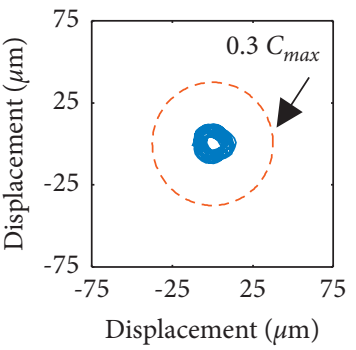

(c)

Figure 14: Test results of rotor axis trajectory. (a) Axis trajectory at A. (b) Axis trajectory at B. (c) Axis trajectory at C.

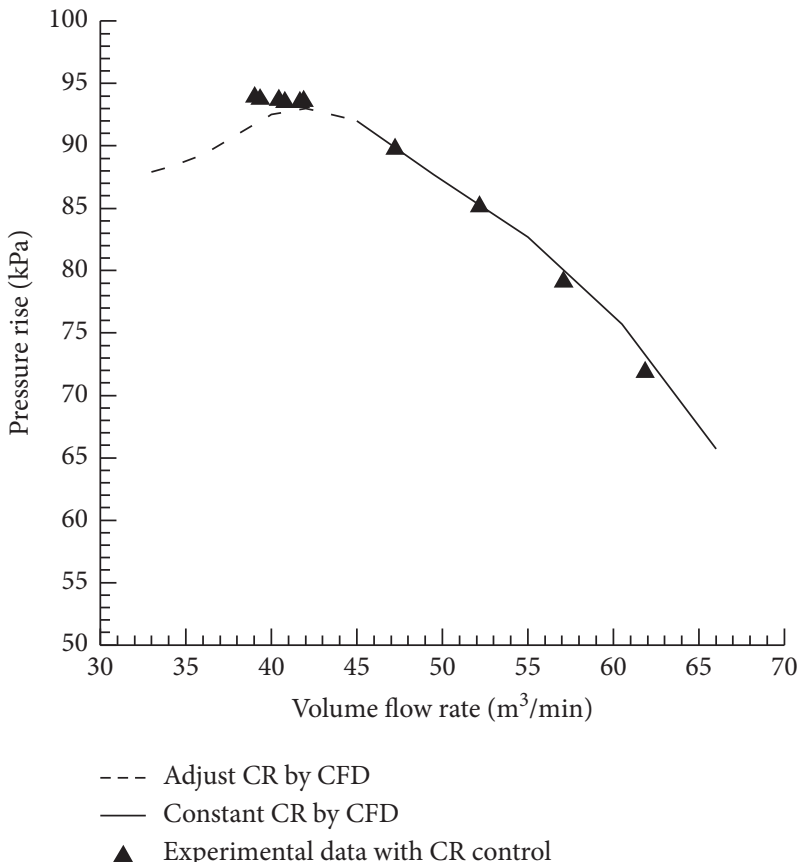

(a)

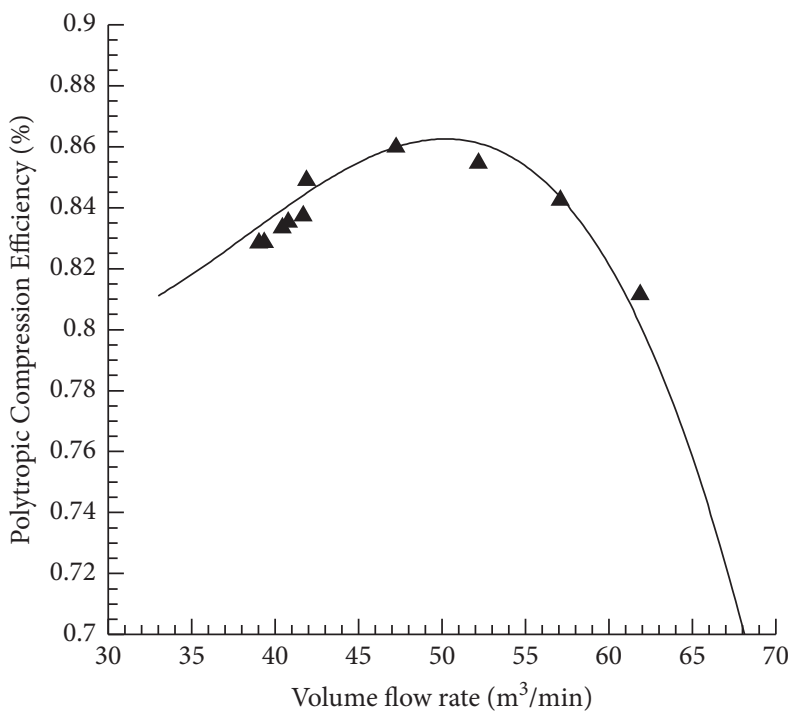

- Experimental data CFD-results

(b)

Figure 15: Test results of flow, pressure, and efficiency under the clearance control law. (a) Pressure ratio over volume flow rate. (b) Polytropic compression efficiency over flow rate.

\section{Conclusions}

The magnetic levitation blower can adjust the tip clearance value during operation to optimize the efficiency of the impeller and significantly widen the surge margin when it deviates from the design point. This paper adopted CFD analysis and experimental verification to study the performance and fluid flow state of the impeller under six different tip clearances at different speeds. After that, an axial clearance control law based on the blower flow rate and pressure was proposed. Then, the control law was tested by experiments. The conclusions of this paper are as follows:

(1) The efficiency and pressure ratio of the impeller decreases linearly with the increase of the tip clearance. Within CR $2.61 \%$ to $7.83 \%$, the pressure 
ratio drops by 0.02 for every $1 \%$ increase in CR and the polytropic compression efficiency decreases by $0.5 \%$.

(2) The surge margin of the impeller enlarges with the augment of the tip clearance mainly because the clearance flow relieves the leading-edge separation under the low flow rate. The simulation results show that, within the scope of the study, for every $1 \%$ increase in CR, the surge margin would increase by $7.8 \%$.

(3) Through active control, under the test clearance control range of $0.3 \mathrm{~mm}-0.7 \mathrm{~mm}$, the surge margin of the blower increases from $26 \%$ to $59 \%$, the surge flow rate at the rated speed reduces from $44 \mathrm{~m}^{3} / \mathrm{min}$ to $36 \mathrm{~m}^{3} / \mathrm{min}$ while the polytropic efficiency remains unchanged.

(4) Bearings with active control can ensure that the impeller obtains the corresponding performance at the design point. When it deviates from the design point, the working range is expanded by adding the tip clearance so that the same impeller can be used in more scenarios which significantly reduce the period of development of the impeller.

\section{Data Availability}

The data used to support the findings of this study are available from the corresponding author upon request.

\section{Conflicts of Interest}

The authors declare that they have no conflicts of interest regarding the publication of this paper.

\section{References}

[1] Y. Senoo, "Pressure loss due to the tip-clearance of impeller blades in centrifugal and axial blowers," Transactions of the Japan Society of Mechanical Engineers, vol. 108, 1984 Lecture Ser. 1984-07).

[2] Y. Senoo and M. Ishida, "Deterioration of compressor performance due to tip clearance of centrifugal impellers," Journal of Turbomachinery, vol. 109, no. 1, pp. 55-61, 1987.

[3] Z. Liu, Y. Ping, and M. Zangeneh, "On the nature of tip clearance flow in subsonic centrifugal impellers," Science China Technological Sciences, vol. 56, no. 9, pp. 2170-2177, 2013.

[4] J. Galindo, A. Tiseira, R. Navarro, and M. A. López, "Influence of tip clearance on flow behavior and noise generation of centrifugal compressors in near-surge conditions," International Journal of Heat and Fluid Flow, vol. 52, pp. 129-139, 2015.

[5] M. Hewkin-Smith, G. Pullan, S. D. Grimshaw, E. M. Greitzer, and Z. S. Spakovszky, "The role of tip leakage flow in spiketype rotating stall inception," Journal of Turbomachinery, vol. 141, no. 6, 2019.

[6] L. M. Du and Y. Z. Gong, "Prediction and analysis of performance of a centrifugal compressor with casing treatment under multiple working conditions," Applied Mechanics and Materials, vol. 448-453, pp. 3434-3439, 2013.
[7] B.-J. Cha, B.-J. Im, and S.-S. Yang, "A study on the instabilities of the centrifugal compressor with variable diffuser," Transactions of the Korean Society of Mechanical Engineers B, vol. 26, no. 8, pp. 1123-1131, 2002.

[8] K. M. Arthur, H. Basu, and S. Y. Yoon, "Stabilization of compressor surge in systems with uncertain equilibrium flow," ISA Transactions, vol. 93, pp. 115-124, 2019.

[9] D. Sanadgol and E. Maslen, "Effects of actuator dynamics in active control of surge with magnetic thrust bearing actuation," in Proceedings of the IEEE/ASME International Conference on Advanced Intelligent Mechatronics, pp. 1091-1096, Monterey, California, July 2005.

[10] S. Y. Yoon, Z. Lin, and P. Allaire, Control of Surge in Centrifugal Compressors by Active Magnetic Bearings, Springer, Berlin, Germany, 2012.

[11] S. Y. Se Young Yoon, Z. Zongli Lin, and P. E. Allaire, "Experimental evaluation of a surge controller for an AMB supported compressor in the presence of piping acoustics," IEEE Transactions on Control Systems Technology, vol. 22, no. 3, pp. 1215-1223, 2014.

[12] S. Y. Yoon, Z. Lin, W. Jiang, and P. E. Allaire, "Flow-rate observers in the suppression of compressor surge using active magnetic bearings," Journal of Turbomachinery, vol. 135, no. 4, pp. 2775-2784, 2013.

[13] M. Tang, J. Zhou, and H. Cui, "Surge control of the centrifugal compressor with magnetic thrust bearing," Process Automation Instrumentation, vol. 38, no. 5, pp. 15-19, 2017.

[14] X. Guan, J. Zhou, and C. Jin, "Influence of different operating conditions on centrifugal compressor surge control with active magnetic bearings," Engineering Applications of Computational Fluid Mechanics, vol. 13, no. 1, pp. 824-832, 2019.

[15] J. Sun, J. Zhao, and K. Wang, "Online surge detection method based on axial displacement sensor of MSCC," IEEE Sensors Journal, vol. 19, no. 15, pp. 6029-6036, 2019.

[16] X. Ma, S. Zheng, and K. Wang, "Active surge control for magnetically suspended centrifugal compressors using a variable equilibrium point approach," IEEE Transactions on Industrial Electronics, vol. 66, no. 12, pp. 9383-9393, 2019.

[17] X. Han, G. Liu, B. Chen, and S. Zheng, "Surge disturbance suppression of AMB-rotor systems in magnetically suspension centrifugal compressors," IEEE Transactions on Control Systems Technology, 2021.

[18] A. Bonfitto, L. M. Castellanos, A. Tonoli, and N. Amati, "Offset-free model predictive control for active magnetic bearing systems," Actuators, vol. 7, no. 3, 2018.

[19] L. M. Castellanos Molina, A. Bonfitto, and R. Galluzzi, "Offset-Free Model Predictive Control for a cone-shaped active magnetic bearing system," Mechatronics, vol. 78, Article ID 102612, 2021.

[20] X. Liu and L. Zhao, "Approximate nonlinear modeling of aircraft engine surge margin based on equilibrium manifold expansion," Chinese Journal of Aeronautics, vol. 25, no. 5, pp. 663-674, 2012.

[21] ISO 14839-2, Mechanical Vibration-Vibration of Rotating Machinery Equipped with Active Magnetic Bearings-Part 2: Evaluation of Vibration, ISO 14839-2, 2004. 